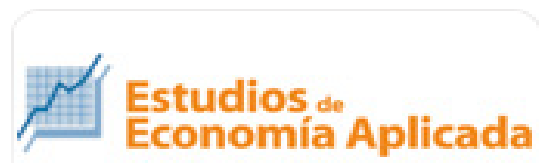
Ectudios de Aplicada

\section{Estudios de Economía Aplicada}

ISSN: 1133-3197

secretaria.tecnica@revista-eea.net

Asociación Internacional de Economía

Aplicada

España

NÚÑEZ MORA, J. ANTONIO; SEGUNDO VALDÉS, ALEJANDRO; CRUZ GALLEGOS, J. LUIS DE LA

Procesos Poisson-Gaussianos para el análisis de rendimientos en el mercado accionarial en México

Estudios de Economía Aplicada, vol. 26, núm. 2, agosto, 2008, pp. 1-15

Asociación Internacional de Economía Aplicada

Valladolid, España

Disponible en: http://www.redalyc.org/articulo.oa?id=30113187014

- Cómo citar el artículo

- Número completo

- Más información del artículo

Página de la revista en redalyc.org

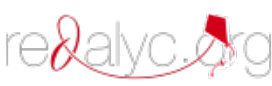

Sistema de Información Científica

Red de Revistas Científicas de América Latina, el Caribe, España y Portugal

Proyecto académico sin fines de lucro, desarrollado bajo la iniciativa de acceso abierto 


\title{
Procesos Poisson-Gaussianos para el análisis de rendimientos en el mercado accionarial en México
}

\author{
J. ANTONIO NÚÑEZ MORA \\ Departamento de Contabilidad y Finanzas \\ TECNOLÓGICO DE MONTERREY, CAMPUS CIUDAD DE MÉXICO \\ ALEJANDRO SEGUNDO VALDÉS \\ Departamento de Economía \\ UNIVERSIDAD AUTÓNOMA METROPOLITANA, UNIDAD AZCAPOTZALCO \\ J. LUIS DE LA CRUZ GALLEGOS \\ Departamento de Finanzas y Economía \\ TECNOLÓGICO DE MONTERREY, CAMPUS ESTADO DE MÉXICO \\ e-mail: janm@itesm.mx
}

\section{RESUMEN}

En la investigación de finanzas y economía es ampliamente reconocido que variables como los rendimientos pueden presentar comportamientos discontinuos. En este sentido, el presente artículo realiza la estimación de los rendimientos para un grupo de acciones pertenecientes a la Bolsa Mexicana de Valores, utilizando para ello procesos de saltos Poisson-Gaussianos y de tipo ARCH, y empleando la aproximación de Das (1998 y 2002) para generar una función de verosimilitud que asegure una estimación robusta. Con base a la metodología planteada, se encuentra que los procesos con saltos capturan características de los datos mexicanos que no siempre son obtenidos mediante la aplicación de los modelos de difusión.

Palabras clave: Saltos; difusión; ARCH; gaussianos; rendimientos.

\section{Analysis of Returns in the Mexican Stock Market through Poisson-Gaussian Processes}

\section{ABSTRACT}

In the financial and economic research, it is known that several financial variables, like returns, can move in a discontinuous manner. This paper proposes and estimates Poisson Gaussian jump and ARCH processes in returns for a set of equities in the Mexican Stock Market. To get the log likelihood, we applied an approximation developed by Das (1998 and 2002). We found that the jump processes capture characteristics of the Mexican data of several equities which would not be captured by diffusion models.

Keywords: Jumps; Diffusions; ARCH; Gaussian; Yields.

Clasificación JEL: C13, C22.

Artículo recibido en septiembre de 2007 y aceptado para su publicación en febrero de 2008 . Artículo disponible en versión electrónica en la página www.revista-eea.net, ref. อ-26213. 


\section{INTRODUCCIÓN}

Durante las últimas décadas, particularmente desde los desarrollos de Fama (1965), la literatura especializada ha señalado que los valores de las series financieras siguen una evolución estocástica, procesos que de acuerdo con Karlin y Taylor (1975) son gobernados con las leyes de probabilidad. En general, desde los desarrollos de Bachelier (1900) y Osborne (1959) la teoría financiera que se ha elaborado para describir la dinámica de los activos y sus rendimientos se encuentra relacionada con los procesos de difusión, en donde uno de los supuestos fundamentales es que los valores de las series tienen una distribución normal. No obstante, a partir de los estudios de Barndorff-Nielsen (1977) diversos trabajos empíricos han mostrado que no es posible sostener la hipótesis de normalidad, fundamentalmente por la evidencia citada por Das (1997) sobre el hecho de que la distribución de los datos exhibe colas largas y "sonrisas" pronunciadas, las cuales son características típicas de series financieras con exceso de curtosis y que por consecuencia no pueden ser modeladas asumiendo que existe una distribución normal. De acuerdo a Kupiec (1995), esto último es una de las mayores violaciones a los supuestos sobre la dinámica de las series financieras, motivo por el cual es necesario estimar su dinámica mediante metodologías cuantitativas que aproximen de una mejor forma la distribución empírica de las series bajo estudio.

En el presente artículo se busca superar dicho problema mediante la aplicación de los procesos del tipo Poisson-Gaussianos, en donde se entiende como modelo Gaussiano a aquel que trata de explicar la evolución estocástica de una variable por medio de los procesos de difusión más utilizados. Por ejemplo, en un modelo Gaussiano los movimientos brownianos generan la estructura de información $F_{t}=\sigma\left(B_{s}, s \leq t\right)$, donde $B_{s}$ es un vector de movimientos brownianos estándar. Debe establecerse que las estructuras de información modeladas de esta manera no permiten la existencia de eventos que sean sorpresivos; la evolución de la variable aleatoria relevante mantendrá un comportamiento relativamente suave, sin grandes cambios y sin saltos en su evolución temporal. No obstante, las investigaciones empíricas contemporáneas desarrollada por Chan, Karolyi, Longstaff y Sanders(1992), Brenner, Harjes y Corner(1994), Das(1994), Heston (1995) y Ait-Sahalia (1995) apuntan a que en algunas ocasiones ello es insuficiente para capturar correctamente la dinámica de variables financieras como los rendimientos, por lo que se debe recurrir a la incorporación de discontinuidades en los procesos estocásticos que describan mejor la trayectoria de las variables. Una forma de capturar el exceso de curtosis de las series financieras es mediante la aplicación de modelos que permitan incorporar explícitamente los saltos en la evolución estocástica de las variables. De manera particular, el comportamiento de algunas variables financieras ha sido estudiado tanto a través de modelos tipo Poisson, como por los métodos de series de tiempo tipo ARCH desarrollados por Gouriéroux (1997) y otros investigadores.

En este sentido, es útil mencionar el caso de la estructura temporal de las tasas de interés, aplicaciones financieras en donde existen formulaciones ampliamente conocidas y fundamentales para la valuación de bonos y derivados. En su forma 
original, y más utilizada, los modelos sobre tasas de interés utilizan una estructura eminentemente Gaussiana. No obstante, los desarrollos de nueva generación como los de Jarrow y Turnbull (1995); Das (1998 y 2002); Segundo (2002) y Shönbucher (2003) se han concentrado en la descripción de una trayectoria estocástica Gaussiana que a su vez incorpora saltos en las tasas de interés, en donde los trabajos de Das (1998 y 2002) y Johannes (2000) son una muestra de que también se ha dotado de un contenido empírico al trabajo teórico, obteniéndose con ello muy buenos resultados en la valuación de deuda corporativa.

Siguiendo estas líneas de investigación, debe mencionarse que también otras variables financieras son susceptibles de ser modeladas por medio de modelos Poisson-Gaussianos, por lo que para el presente estudio se aplican dichas metodologías en el análisis de los rendimientos de acciones que cotizan en la Bolsa Mexicana de Valores (BMV). En este sentido, es importante hacer una diferenciación que justifica la utilización de este tipo de modelos para el caso de los rendimientos: en los mercados accionarios la llegada e incorporación de la información no se realiza de manera continua, ni en "paquetes" de información (Quantos) de la misma calidad y cantidad informativa. Además, debe resaltarse que aunque la trayectoria de los rendimientos de las acciones es aleatoria, no es suficiente emplear la típica descripción Gaussiana. Finalmente, Balduzzi et al. (1998) y Green (1998) han examinado distintas fuentes de información que pueden llegar a influir sobre el mercado financiero, y en donde las noticias sobre la política económica, la situación laboral de los países, el movimiento de los índices en otros mercados accionarios internacionales, principalmente, afectan las decisiones de compra y venta de las acciones; y en consecuencia perturban el precio y los rendimientos de las mismas.

Evidentemente, en la valuación de los derivados financieros sobre acciones la literatura reciente se ha concentrado en mejorar el artículo de Black \& Scholes (1973), en donde la modelación de los precios se da a partir del Movimiento Geométrico Browniano (GBM). La razón esencial de la búsqueda de nuevas formas de análisis se basa en el hecho de que diversos estudios empíricos han mostrado la existencia de una subvaluación de los derivados, como en el caso de Black (1975), la cual es corregida cuando se incluyen saltos en la dinámica del precio de las acciones, y en donde los trabajos de Merton (1976), Ball y Torous (1985), Jorion (1988), Bakshi, Cao y Chen (1997) representan destacados ejemplos de ello.

Por tanto, conocer la dinámica que siguen los precios o rendimientos de las acciones es fundamental para proceder a evaluar correctamente los derivados o instrumentos financieros que dependan de ellos. Para el caso de los activos que cotizan en la BMV, Ramírez (2004), Trejo, Núñez y Lorenzo (2006), ya han demostrado que generalmente los rendimientos de los mismos, así como sus incrementos presentan exceso de curtosis, ante lo cual es necesario examinarlos de una manera distinta a la empleada habitualmente. En consecuencia, el objetivo central de la presente investigación es el de analizar empíricamente la evolución de los rendimientos de un grupo de acciones que cotizan en la Bolsa Mexicana de Valores, mediante los modelos del tipo Poisson-Gaussiano, Poisson-Gaussiano- 
ARCH y ARCH-Gaussiano. Con este propósito, se utiliza la base teórica desarrollada por Das (1998 y 2002) para generar una función de verosimilitud que asegure una estimación robusta. El periodo de estudio abarca del 4 de enero de 1994 al 23 de marzo del 2004 con una frecuencia diaria. El resto del trabajo se divide en: 2. El modelo, 3.Aplicación empírica y 4.Conclusiones.

\section{EL MODELO}

Si se supone un proceso estocástico general que incluye un proceso de difusión y una descripción de las posibles discontinuidades en la trayectoria de los rendimientos accionarios, en donde los rendimientos de las acciones se calculan como la diferencia de los logaritmos naturales de los precios, un proceso estocástico con reversión a la media para el rendimiento de los activos puede describirse mediante la ecuación 1:

$$
d r=k(\theta-r) d t+v d z+J d \pi(h)
$$

En esta ecuación $r$ representa el rendimiento de la acción, $\theta$ es el parámetro de tendencia central para $r$, en tanto que $k$ indica la tasa de reversión del rendimiento $r$. Como es natural, $v$ representa la desviación estándar, y $z$ es un movimiento browniano estándar. Además $\pi$ constituye un proceso de Poisson que describe la llegada de los saltos aleatorios; $h$ es un parámetro de la frecuencia de los saltos y $J$ representa al tamaño de los saltos. La descripción de $J$ es bastante general, puede ser constante o se puede obtener de una distribución de probabilidad específica. Se supone que el proceso de difusión $(z)$ y el proceso Poisson $(\pi)$ son estadísticamente independientes. También $z$ y $\pi$ son independientes del proceso $J$. En este punto es relevante hacer la observación de que la ecuación [1] es utilizada por Das para describir la evolución estocástica de la tasa de interés. Sin embargo, debe notarse que la forma de la ecuación permite la existencia de valores negativos para $r$. Pero si $r$ es una tasa de interés, entonces no tiene sentido económico ni financiero la expresión anterior. En el caso del presente desarrollo, $r$ representa los rendimientos accionarios, variables que si pueden tener valores positivos o negativos sin incurrir en una contradicción económica.

Para el proceso descrito por la ecuación [1], es posible obtener la función característica para el intervalo $\left(t_{0}-T\right)$ de la forma $F[r(t), T ; s]=\exp [A(T ; s)+r B(T ; s)]$. Con la función característica anterior es posible conseguir los momentos y las funciones de densidad de probabilidad para cualquier distribución con saltos. La novedad de este análisis consiste en que los momentos pueden ser derivados sin necesidad de identificar previamente la función característica de forma cerrada. Como es conocido, los procesos del tipo Poisson-Gaussianos necesitan obtener la densidad de probabilidad de transición condicional de procesos de difusión con salto. Para hacer esto, se aplica la inversión de Fourier de la función característica $F[r(t), T ; s]$, en donde $t$ representa el tiempo presente, y $\tau$ es el intervalo de tiempo, 
tal que $\tau=T-t$. La inversión de Fourier de $F[r(t), T ; s]$ tiene por resultado la función de densidad de transición:

$$
f[r(t+\tau) / r(t)]=\frac{1}{\pi} \int_{0}^{\infty} \operatorname{Re}[\exp (-i s r(t+\tau)) F[r(t), \tau ; s]] d s
$$

La estimación se realiza por medio del método de máxima verosimilitud, utilizando para este propósito los rendimientos en tiempo discreto de las acciones. Si el intervalo de tiempo entre las observaciones se escribe mediante el símbolo $\Delta$, entonces, la función de verosimilitud $(L)$ que es maximizada se escribe como:

$$
\begin{array}{ll}
L=\quad \operatorname{Max} & \sum_{t=0}^{T-1} \log (f[r(t+\Delta)] \mid r(t))
\end{array}
$$

La integral de Fourier puede obtenerse numéricamente con el cálculo de la integral involucrada en la ecuación [3]. Las segundas derivadas numéricas de la función de verosimilitud evaluadas en el conjunto óptimo $\Omega^{*}=\left\{\kappa^{*}, \theta^{*}, v^{*}, h^{*},\left\{E\left[j^{n}\right]^{*}\right\}\right\}$ proporciona la matriz hessiana de la forma $\frac{\partial^{2} L}{\partial \Omega \partial \Omega^{\prime}}$. Finalmente, los errores estándar se pueden calcular como $\sqrt{\operatorname{diag}\left(-X^{-1}\right)}$. En este artículo se estima una aproximación discreta del modelo anterior, en donde los saltos aleatorios tienen distribución normal.

En particular se supone que los rendimientos de las acciones siguen un proceso de difusión con saltos. Para ello se toma la aproximación de los saltos aleatorios de Bernoulli propuesta por Ball y Torous (1983). La idea sobre la cual se construye esta modelación consiste en suponer que en cada intervalo de tiempo considerado únicamente hay dos posibilidades: a) ocurre un salto y, b) no ocurre un salto. Como se sabe, en el límite, un proceso Bernoulli es gobernado por una distribución de Poisson. Con esta idea, se construye una aproximación a la función de verosimilitud para un modelo Poisson-Gaussiano con una mezcla de distribuciones normales. La aproximación Bernoulli se obtiene definiendo una variable indicadora $Y_{i}$, para $i=1,2, \ldots, n$. La variable $Y_{i}$ tomará el valor de uno si es que ocurre el salto, y $Y_{i}$ vale cero si no hay salto. Como $\Delta t=T / N$ para las series de tiempo, entonces

$$
\begin{aligned}
& \operatorname{Pr}\left[Y_{i}=0\right]=1-h \Delta t+O(\Delta t) \\
& \operatorname{Pr}\left[Y_{i}=1\right]=h \Delta t+O(\Delta t) \\
& \operatorname{Pr}\left[Y_{i}>1\right]=O(\Delta t)
\end{aligned}
$$

Si se define la variable $M=\sum_{i=1}^{N} Y_{i}$, entonces $M$ tiene una distribución binomial. Y para un número $x$ de ocurrencias de salto tenemos $\lim _{N \rightarrow \infty} \operatorname{Pr}[M=x]=e^{-h T}(h T)^{x} / x !$. 
Ball y Torous (1983) demuestran que la aproximación es estable, manejable numéricamente y convergente. Ahora, el proceso del rendimiento de las acciones descrito en la ecuación [1] puede aproximarse en tiempo discreto como:

$$
\Delta r=k(\theta-r) \Delta t+v \Delta z+J\left(\mu, \sigma^{2}\right) \Delta \pi(q)
$$

donde:

- $v^{2}=$ varianza anualizada del shock gaussiano.

- $\Delta z=$ término del shock de una normal estándar.

$J\left(\mu, \sigma^{2}\right)=$ shock de salto, que se distribuye normalmente con media $\mu$ y varianza $\sigma^{2}$. $\Delta \pi(q)=$ incremento Poisson discreto, aproximado por la distribución de Bernoulli con el parámetro $q$.

Las probabilidades de transición para los rendimientos de las acciones siguen un proceso Poisson-Gaussiano, para $(s>t)$, de la siguiente forma (Das, 1998 y 2002):

$$
\begin{aligned}
f[r(s) / r(t)]= & q \exp \left[\frac{-(r(s)-r(t)-\kappa(\theta-r(t)) \Delta t-\mu)^{2}}{2 v^{2} \Delta t+\sigma^{2}}\right] \frac{1}{\sqrt{2 \pi\left(v^{2} \Delta t+v^{2}\right)}}+ \\
& +(1-q) \exp \left[\frac{-(r(s)-r(t)-\kappa(\theta-r(t)) \Delta t)^{2}}{2 v^{2} \Delta t}\right] \frac{1}{\sqrt{2 \pi v^{2} \Delta t}}
\end{aligned}
$$

En esta ecuación $q$ es aproximado como $q=h \Delta t+O(\Delta t)$. En realidad la ecuación [5] es la aproximación a la verdadera densidad Poisson-Gaussiana a través de una mezcla de distribuciones normales. Para la estimación se debe llevar a cabo la siguiente optimización:

$$
\underset{\left\{\kappa, \theta, v, \mu, \sigma^{2}, q\right\} \quad \sum_{t=1}^{T} \log (f[r(s) \mid r(t)])}{\operatorname{Max}}
$$

La optimización anterior se realiza por medio del método de máxima verosimilitud.

\section{APLICACIÓN EMPÍRICA}

En el presente documento se utilizaron nueve series de tiempo correspondientes a los rendimientos obtenidos por acciones de empresas que cotizan en la BMV. El periodo que abarcan las observaciones de rendimientos va del 4 de enero de 1994 al 23 de marzo de 2004, es decir, se tienen 2.560 observaciones para cada una de las series escogidas. Las nueve acciones seleccionadas pertenecen a empresas emisoras con una alta bursatilidad, y que además constituyen una muestra representativa del mercado accionario mexicano. Con la elección de dicha muestra también se evitan los problemas estadísticos típicos de las series de rendimientos que fue- 
ron señalados por Lo y McKinlay (1990), y que generalmente son conocidos como asincronía de las transacciones. Para el caso de las acciones bajo estudio, y en diferentes periodos de tiempo, Segundo y Núñez, 2004 ya han demostrado que las acciones utilizadas en el presente trabajo no generan autocorrelación espuria debido al fenómeno de asincronía. En consecuencia con lo anterior, y siguiendo la metodología propuesta, es necesario observar el comportamiento general de la muestra de rendimientos escogido. En la Tabla 1 se reportan los cuatro primeros momentos de las series, y el estadístico de Jarque-Bera (1980), junto con su valor probabilidad ( $p$-value).

TABLA 1

Momentos de los Rendimientos de las Acciones.Valores Máximo y Mínimo en el periodo de estudio.

\begin{tabular}{|l|c|c|c|c|r|r|}
\hline & Media & Varianza & Sesgo & Curtosis & \multicolumn{1}{|c|}{ JB } & p-value \\
\hline Alfa & 0.000804 & 0.000737 & 0.357683 & 9.276071 & 5410.03 & 0.0000 \\
Carso & 0.000210 & 0.000780 & -2.48651 & 46.48194 & 248689 & 0.0000 \\
Kimberly & 0.000560 & 0.000500 & 0.295470 & 11.86365 & 9955.1 & 0.0000 \\
Com Mex & 0.000400 & 0.000730 & 0.172000 & 11.23980 & 9044.06 & 0.0000 \\
ICA & -0.00036 & 0.001220 & 0.280290 & 14.83060 & 807276.9 & 0.0000 \\
Coca Cola & 0.000870 & 0.000630 & 0.028730 & 7.447550 & 163968.1 & 0.0000 \\
CEMEX & 0.000510 & 0.000660 & -0.06308 & 10.98266 & 8490.73 & 0.0000 \\
APASCO & 0.000630 & 0.000640 & 0.561060 & 9.967450 & 5922.32 & 0.0000 \\
Bimbo & 0.000500 & 0.000490 & 0.173180 & 6.488800 & 857805472 & 0.0000 \\
\hline
\end{tabular}

Fuente: Elaboración propia con información de la base de datos de Reuters.

Como se puede observar en los estadísticos presentados, la información de las series sugiere que los rendimientos de las acciones no se distribuyen de manera normal; principalmente porque en todos los casos hay un exceso de curtosis. En este sentido dichos resultados son consistentes con los estudios de Trejo, Núñez y Lorenzo (2006) y Ramírez (2004), quienes ya han demostrado que los rendimientos de las acciones en la Bolsa Mexicana de Valores no siguen una distribución normal. En particular, para el periodo de análisis que se está considerando, y para el presente grupo de acciones, Segundo (2006) también ha encontrado que los rendimientos diarios no se distribuyen normalmente. De hecho, gran parte de la agenda de investigación previa se ha centrado en la búsqueda de distribuciones que ajusten mejor los datos empíricos, especialmente las relacionadas a los trabajos de Eberlein y Keller, (1995), Bibby y Sørensen (1995), Barndorff-Nielsen (1995), y para el caso Mexicano Ramírez (2004), Trejo, Núñez y Lorenzo (2006).

En la Tabla 2 se muestran los cuatro primeros momentos de la serie de los incrementos en los rendimientos de las acciones; se indica el valor mínimo y el valor máximo de toda la serie. Los datos señalan que en este caso el exceso de curtosis que mostraban las series originales también se encuentra presente. La existencia de 
leptocurtosis en las series de los cambios en los rendimientos sugiere que la evolución de los mismos puede explicarse por la presencia de saltos en su evolución estocástica en el tiempo.

TABLA

Momentos de los Incrementos de los Rendimientos de las Acciones.

Valores Máximo y Mínimo en el periodo de estudio.

\begin{tabular}{|l|r|r|r|r|r|r|}
\hline & \multicolumn{1}{|c|}{ Media } & \multicolumn{1}{c|}{ Varianza } & \multicolumn{1}{c|}{ Sesgo } & \multicolumn{1}{c|}{ Curtosis } & \multicolumn{1}{c|}{ Mínimo } & \multicolumn{1}{c|}{ Máximo } \\
\hline Alfa & -0.000006 & 0.001261 & -0.041112 & 7.33005 & -0.214674 & 0.201641 \\
Carso & -0.000013 & 0.001427 & 0.677166 & 32.918176 & -0.500673 & 0.510724 \\
Kimberly & -0.000007 & 0.000959 & 0.779813 & 15.385626 & -0.233846 & 0.355733 \\
Com Mex & -0.000014 & 0.001436 & -0.253308 & 15.549204 & -0.436521 & 0.318991 \\
ICA & 0.000018 & 0.002306 & -0.276607 & 11.143128 & -0.430201 & 0.362616 \\
Coca Cola & 0.000004 & 0.001205 & 0.124012 & 6.877401 & -0.237096 & 0.192183 \\
CEMEX & -0.000011 & 0.001187 & 0.869023 & 16.388507 & -0.212858 & 0.399981 \\
APASCO & -0.000002 & 0.001271 & 0.568987 & 14.276639 & -0.239400 & 0.343622 \\
Bimbo & -0.0000004 & 0.000925 & 0.072345 & 6.645250 & -0.175300 & 0.200582 \\
\hline
\end{tabular}

Fuente: Elaboración propia con información de la base de datos de Reuters.

Para verificar si existen saltos en las trayectorias de los rendimientos se han estimado dos modelos. El primero de ellos consiste en estimar la versión discreta de la ecuación que describe el proceso de difusión con saltos, es decir, la ecuación [4]. Para hacer esto, se resuelve por medio del método de máxima verosimilitud la expresión [6]. Este modelo es Poisson-Gaussiano. El segundo modelo es una extensión del modelo Poisson-Gaussiano al que se le agrega un proceso ARCH(1) para la descripción de la evolución de la varianza del componente Gaussiano de la ecuación [4]. Este es un modelo ARCH-Poisson-Gaussiano. La varianza de la parte Gaussiana se comporta de acuerdo a la siguiente expresión:

$$
v(s+\Delta t)^{2}=a_{0}+a_{1}[r(s)-E\{r(s)\}]^{2} .
$$

Primeramente, en la Tabla 3 se reportan los resultados del modelo PoissonGaussiano. El análisis toma observaciones de rendimientos diarios de acciones de empresas durante el lapso de tiempo bajo estudio. Para la estimación se incorpora la función de densidad de transición. La versión discreta del modelo sigue la ecuación $\Delta r=\kappa(\theta-r) \Delta t+v \Delta z+J\left(\mu, \sigma^{2}\right) \Delta \pi(q)$. La variable $q$ representa la probabilidad de salto del rendimiento en el período $\Delta$. $q$ es análoga a la variable $h$ del modelo en tiempo continuo y entre paréntesis se encuentran reportados los errores estándar. 


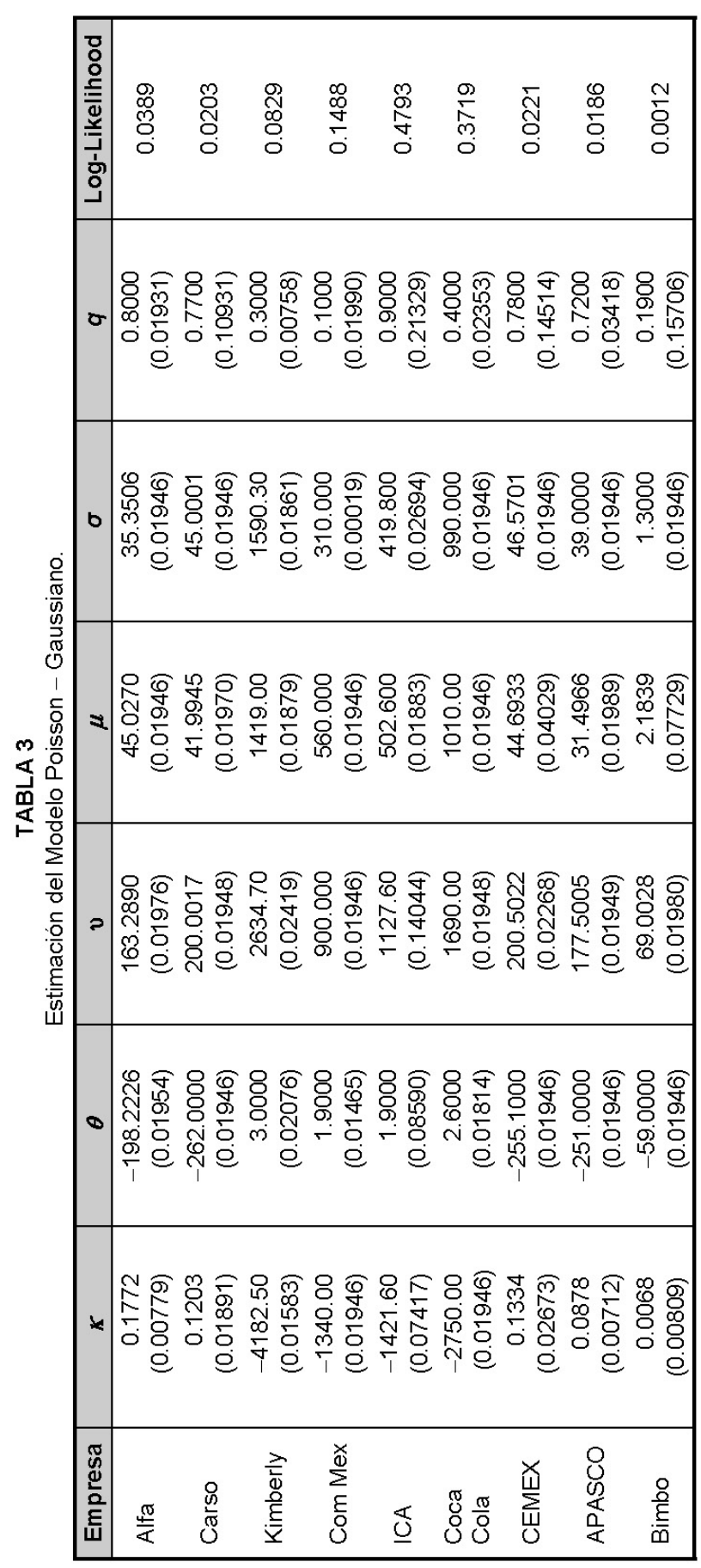

Estudios de Economía Aplicada, 2008: 1-15, Vol. 26-2 


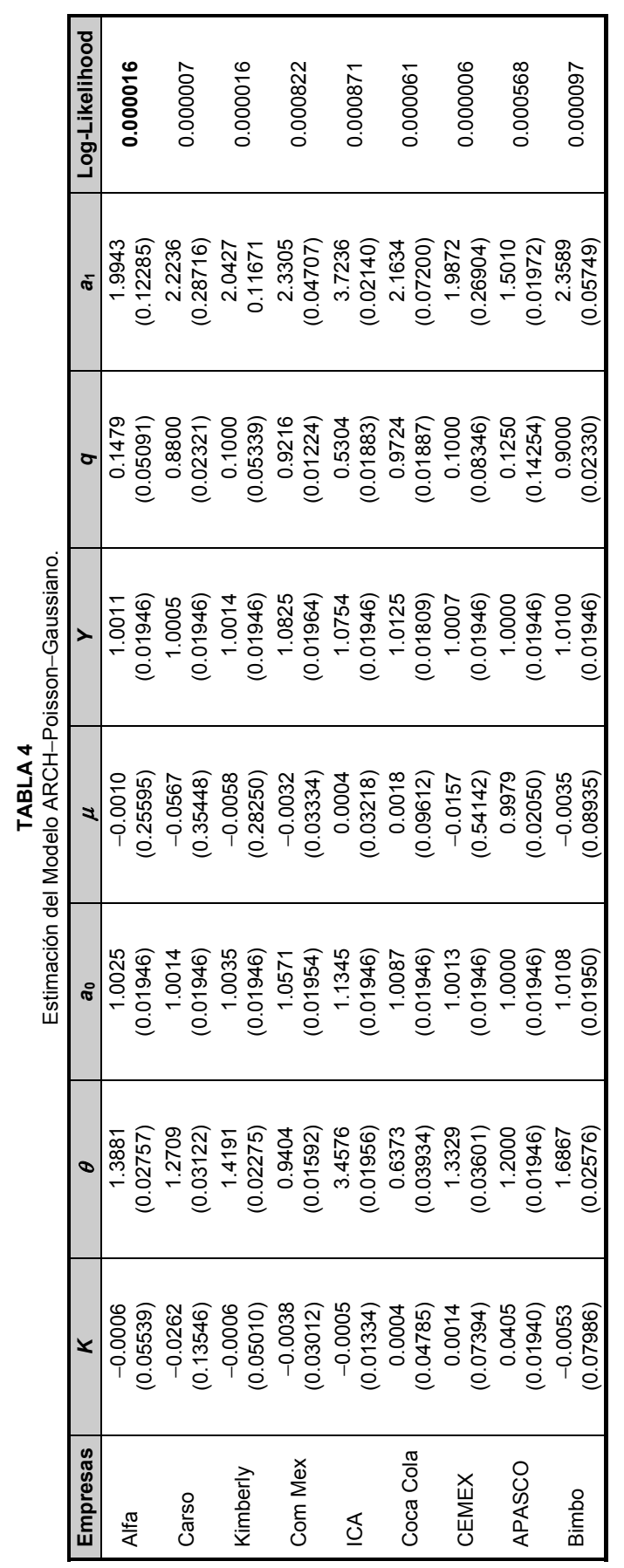

Estudios de Economía Aplicada, 2008: 1-15, Vol. 26-2 
En la Tabla 4 se citan los resultados del modelo ARCH-Poisson-Gaussiano para el mismo período de tiempo. Para la estimación se incorpora la función de densidad de transición. En este caso la versión discreta del modelo sigue tiene la siguiente especificación:

$$
\begin{gathered}
\Delta r=k(\theta-r) \Delta t+v \Delta z+J\left(\mu, \sigma^{2}\right) \Delta \pi(q) \\
v(s+\Delta t)^{2}=a_{0}+a_{1}[r(s)-E\{r(s)\}]^{2}
\end{gathered}
$$

Entre paréntesis están reportados los errores estándar.

En la Tabla 5 se aplica la prueba Dickey-Fuller aumentada para inferir la estacionariedad de las series, y explorar en modelo ARCH-Gaussiano cuyos resultados se muestran en la Tabla 6 (entre paréntesis están reportados los errores estándar). Para la estimación se incorpora la función de densidad de transición. La versión discreta del modelo sigue tiene la siguiente especificación:

$$
\begin{aligned}
& \Delta r=k(\theta-r) \Delta t+v \Delta z+v \Delta z+J\left(\mu, \sigma^{2}\right) \Delta \pi(q) \\
& v(s+\Delta t)^{2}=a_{0}+a_{1}[r(s)-E\{r(s)\}]^{2}
\end{aligned}
$$

El análisis toma observaciones de rendimientos diarios de acciones de empresas durante el periodo que comienza el 4 de enero de 1994, y se extiende hasta 23 de marzo de 2004.

TABLA 5

Prueba de Raíces Unitarias (ADF).

\begin{tabular}{|l|c|c|c|}
\hline \multicolumn{4}{|c|}{ Raíz unitaria sobre los rendimientos } \\
\hline \multicolumn{1}{|c|}{ Activo } & estadístico $t$ & Significancia 1\% & $p$-value \\
\hline Alfa & -36.96 & -3.96 & 0.0000 \\
Apasco & -50.46 & -3.96 & 0.0000 \\
Bimbo & -31.17 & -3.96 & 0.0000 \\
Carso & -46.96 & -3.96 & 0.0000 \\
Cemex & -45.83 & -3.96 & 0.0000 \\
Coca Cola & -14.40 & -3.96 & 0.0000 \\
Comercial Mexicana & -49.98 & -3.96 & 0.0000 \\
ICA & -55.22 & -3.96 & 0.0000 \\
Kimberly & -48.11 & -3.96 & 0.0000 \\
\hline
\end{tabular}




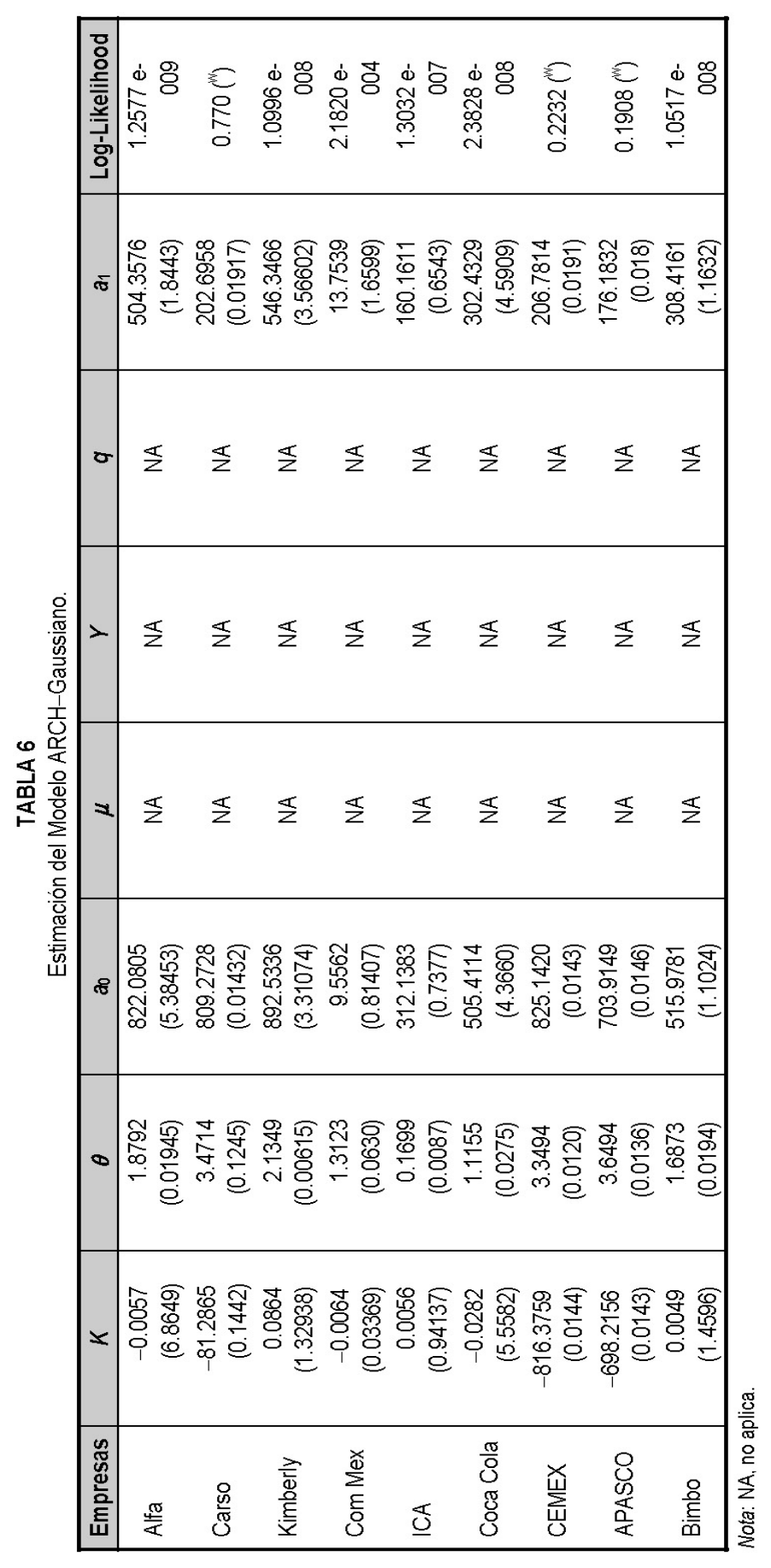

Estudios de Economía Aplicada, 2008: 1-15, Vol. 26-2 
Una inspección de los estadísticos muestra que el modelo Poisson-Gaussiano tiene un mejor desempeño que el modelo ARCH-Poisson-Gaussiano. De manera similar también debe observarse que el valor de logaritmo de la verosimilitud es mayor para el caso del modelo Poisson-Gaussiano. La excepción en este se presenta cuando se desarrolla el ARCH, en donde los casos Carso, Cemex y Apasco (Tabla 6) son mejores que el Poisson-Gaussiano respectivo. En el resto de la Tabla 6, los errores de los parámetros son considerablemente más grandes que en los obtenidos con los del modelo Poisson-Gaussiano.

Si se compara con estas dos representaciones, el modelo Gaussiano puro (con el valor de $h=0$ ) no permite describir correctamente la evolución estocástica de las series bajo estudio, tal y como lo había demostrado Das (1998). En términos generales la razón se encuentra en el hecho de que el modelo Gaussiano no reconstruye de manera adecuada el comportamiento de las series de rendimientos ante la aparición de información sorpresiva en los mercados accionarios. Finalmente, debe observarse que el valor estimado de $q$ debe interpretarse como la probabilidad de ocurrencia de un salto en cualquier día. Los resultados reportados en las tablas 3 y 4 para los dos modelos son distintos, no puede hacerse una interpretación directa. Las probabilidades de salto aleatorio diario cambian, a veces dramáticamente, entre un modelo y otro.

\section{CONCLUSIONES}

En el presente estudio se ha argumentado la conveniencia de tomar en cuenta las discontinuidades en la evolución estocástica de los rendimientos de un grupo de acciones representativas que cotizan en la BMV. Se han realizado pruebas empíricas con tres modelos: el Poisson-Gaussiano, el ARCH-Poisson-Gaussiano y el ARCH-Gaussiano. Los resultados obtenidos permiten concluir que el modelo Poisson-Gaussiano tiene un mejor ajuste estadístico y por lo tanto es mejor para representar e interpretar las series de tiempo de rendimientos accionarios de la muestra escogida, aunque debe remarcarse que en tres acciones las verosimilitudes de los ARCH fueron ligeramente mejores. Estos resultados confirman lo que a nivel empírico se ha obtenido en otras investigaciones aplicadas a modelar las estructuras temporales de tasas de interés: las series de tiempo de datos financieros, en su mayoría, no pueden describirse correctamente con modelos puros Gaussianos, fundamentalmente porque al agregar discontinuidades adicionales a los modelos se obtiene una representación más adecuado de la dinámica de los datos, permitiendo con ello la realización de una mejor valuación de los mismos. 


\section{REFERENCIAS BIBLIOGRÁFICAS}

AIT-SAHALIA, Y. (1995): "Testing Continuous-Time Models of the Interest Rate", Review of Financial Studies, $n^{\circ}$ 9, pp. 385-426.

BACHELIER, L. (1900): "Théorie de la spéculation", Annales de l'Ecole Normale Supériore, $\mathrm{n}^{\circ} 17$, pp. 21-86.

BAKSHI, G.; CAO, C. Y CHEN, Z. (1997): "Empirical Performance of Alternative Option Pricing Models", Journal of Finance, $n^{\circ}$ 52(5), pp. 2003-2049.

BALDUZZI, P.; GREEN, T.C. Y ELTON, E. (1998): "Economic News and the Yield Curve: Evidence from the U.S. Treasury Market", Working Paper, New York University.

BALL, C. Y TOROUS, W. (1983): "A Simplified Jump Process for Common Stock Returns", Journal of Financial and Quantitative Analysis, vol. 18, n 1, pp. 53-65.

BARNDORFF-NIELSEN, O. E. (1995): "Normal inverse Gaussian processes and the modeling of stock returns", Research Report 300, Department of Theoretical Statistics, Institute of Mathematics, University of Aarhus.

Bandorff-Nielsen, O.E. (1977): "Infinite Divisibility of the Hyperbolic and Generalized Inverse Gaussian Distributions, Z. Wahrsheinlichkeit-stheorie und Verw., Gebiete, n 38, pp. 309-312.

BIBBY, B. M. Y SØRENSEN, M. K. (1995): "A Hyperbolic Diffussion Model for Stock Prices", Research Report 331, Department of Theoretical Statistics, Institute of Mathematics, University of Aarhus.

BLACK, F. (1975): "Fact and Fantasy in the Use of Options", Financial Analyst Journal, $\mathrm{n}^{\circ} 31$, pp. 36-72.

BLACK, F. Y SCHOLES, M. (1973): "The Pricing of Options and Corporate Liabilities", Journal of Political Economy, $\mathrm{n}^{\circ}$ 81, pp. 637-654.

BALL, C. A. Y TOROUS, W. (1983): "A Simplified Jump Process for Common Stock Returns", Journal of Financial and Quantitative Analysis, n 18(1), pp. 53-65.

BALL, C. A. Y TOROUS, W. (1985): "On Jumps in Common Stock Prices and Their Impact on Call Option Pricing", The Journal of Finance, n 40(1), pp. 155-173.

BRENNER, HARJES, R. Y KRONER K. (1994): "Another Look at Alternative Models of the Short-Term Interest Rate", Merrill Lynch, Wells Fargo and University of Arizona.

CHAN, K.; KAROLYI, G.; LONGSTAFF, F. Y SANDERS, B. (1992): "An Empirical Comparison of Alternative Models of the Short-Term Interest Rate", Journal of Finance, $\mathrm{n}^{\circ} 47$, pp. 1209-1228.

CONT, RAMA Y TANKOV, P. (2004): Financial Modeling with Jump Processes, Chapman \& Hall/CRC Financial Mathematics Series, USA.

DAS, SANJIV R. (1994): "Poisson-Gaussian Processes and the Bond Markets", Working Paper, Harvard University Business School.

DAS, SANJIV R. (1997): "An Efficient Generalized Discrete-time Approach to PoissonGaussian Bond Option Pricing in the Heath-Jarrow-Morton Model, NBER Working Paper Series, $\mathrm{n}^{\circ} 212$.

DAS, SANJIV R. (1998): "Poisson Gaussian Processes and the Bond Markets", NBER Working Paper Series, $\mathrm{n}^{\circ} 6631$.

DAS, SANJIV R. (2002): "The Surprise Element: Jumps in Interest Rates", Journal of Econometrics, $\mathrm{n}^{\circ} 106$, pp. 27-65.

EBERLEIN, E. Y KELLER, U. (1995): "Hyperbolic Distributions in Finance", Bernoulli 1, pp. 281-299.

FAMA, E. (1965):"The behavior of Stock Market Prices", Journal of Business, n 38(1), pp. 34-105.

GREEN, T.C. (1998): "New Releases, Asymmetric Information, and Intraday Price Formation in the Interdealer Market for Treasury Securities", Working Paper, New York University. 
GOURIÉROUX, C. (1997): Arch Models and Financial Applications, Springer Series in Statistics.

HESTON, S. (1995): "A model of Discontinuous Interest Rate Behavior, Yield Curves and Volatility", Working Paper, Washington University.

KARLIN, S. Y TAYLOR H. (1975): A First Course in stochastic Processes, Academic Press.

KUPIEC, P.H. (1995): "Techniques for Verifying the Accuracy of Risk Management Models", The Journal of Derivatives, $\mathrm{n}^{\circ} 3$, pp. 73-84.

JARQUE, C. M. Y BERA, A. (1980): "Efficiency Test for Normality, Heterocedasticity and Serial Independence of Regression Residuals", Economic Letters, n 6, pp. 255-259.

JARROW, R. Y TURNBULL, S. M. (1995): "Pricing Derivatives on Financial Securities Subject to Credit Risk", The Journal of Finance, vol. 50, n 1, pp. 53-86.

JOHANNES, M. S. (2000): A Nonparametric View of the Role of Jumps to Interest Rates, working paper, graduate School of Business, Columbia University.

JORION, P. (1988): "On Jump Processes in the Foreign Exchange and Stock Markets", Review of Financial Studies, $\mathrm{n}^{\circ} 1(4)$, pp. 427-445.

LO, A. Y MACKINLAY, C. (1990): "An Econometric Analysis of Nonsynchronous Trading", Journal of Econometrics, vol. 45, reproduced in: Lo, A. and MacKinlay, C., A NonRandom Walk Down Wall Street, Princeton University Press, pp. 85-113.

MERTON, R. (1975): "An Asymptotic Theory of Growth under Uncertainty", Review of Economic Studies, $\mathrm{n}^{\circ} 42$, pp. 375-393.

OSBORNE, M. (1959): "Brownian Motion in the Stock Market", en Cootner Paul, The Random Character of Stock Market Prices, Cambridge, MIT Press, 1964.

RAMÍREZ, J.C. (2004): "Usos y limitaciones de los procesos estocásticos en el tratamiento de distribuciones de rendimientos con colas gordas", La Revista de Análisis Económico, no 19 (1), pp. 51-76.

SEGUNDO, A. (2002): Valuación de Deuda con Riesgo, Tesis Doctoral, ITESM, México.

SEGUNDO, A. Y NÚÑEZ, J. A. (2004): "La Asincronía de las Transacciones en la Bolsa Mexicana de Valores", Economía: Teoría y Práctica. Nueva Época. n²1. pp. 145-167.

SEGUNDO, A. (2006): La Hipótesis de Caminata Aleatoria en la Bolsa Mexicana de Valores (1994-2004), documento de investigación, UAM, México.

SHÖNBUCHER, P. (2003): Models, Pricing and Implementation, Wiley editions.

TREJO, B.; NÚÑ̃EZ, J. A. Y LORENZO, A. (2006): "Distribución de los Rendimientos del Mercado Mexicano Accionario", Estudios Económicos, vol. 21, n 1, Enero-Junio, El Colegio de México, pp. 85-118. 